\title{
DIRAC MATRICES IN CHIRAL REPRESENTATION AND THE CONNECTION WITH THE ELECTRIC FIELD PARALLEL TO THE MAGNETIC FIELD
}

\section{MATRICES DE DIRAC EN REPRESENTACIÓN QUIRAL Y LA CONEXIÓN CON EL CAMPO ELÉCTRICO PARALELO AL CAMPO MAGNÉTICO}

\author{
H. Torres-Silva ${ }^{1}$ \\ Recibido el 5 de septiembre de 2007, aceptado el 5 de diciembre de 2007 \\ Received: September 5, 2007 Accepted: December 5, 2007
}

\begin{abstract}
RESUMEN
En este trabajo se presenta una expresión de la transformación general de Foldy-Wouthuysen a la representación quiral de las matrices de Dirac interactuando con un campo de fermión. La hipótesis es que a través de la multiplicación de la matriz de Pauli por las ecuaciones quirales de Maxwell en el caso de $\vec{E}=i \eta \vec{H}$, se obtiene la ecuación quiral de Dirac. Esta es la prueba del teorema de que la mecánica de ondas de partícula cuántica representa una electrodinámica especializada.
\end{abstract}

Palabras clave: Transformación de Foldy-Wouthuysen, ecuación quiral de Dirac, electrodinámica.

\section{ABSTRACT}

In this paper we offer an expression of the general Foldy-Wouthuysen transformation in the chiral representation of Dirac matrices interacting with fermion field. Our hypothesis is that through the multiplication of the Pauli matrix and Maxwell's chiral equations in the case of $\vec{E}=i \eta \vec{H}$, one obtains the Dirac's chiral equation. This is the proof of the theorem that the wave mechanics of quantum particles represent a specialized electrodynamic.

Keywords: Foldy-Wouthuysen transformation, chiral Dirac equation, electrodynamics.

\section{CHIRAL DIRAC MATRICES}

The paper offers an expression of the general FoldyWouthuysen transformation in the chiral representation of Dirac matrices interacting with fermion field $\psi(\vec{x}, t)$. The paper $[1,2]$ discuss the theory of interacting quantum fields in the Foldy-Wouthuysen representation [3]. These papers offer, in particular, the relativistic nonlocal Hamiltonian $H_{F W}$ in the form of a series in terms of powers of charge $e$. Quantum electrodynamics in the Foldy-Wouthuysen $(F W)$ representation has been formulated using Halmitonian $H_{F W}$ and some quantum electrodynamics processes have been calculated within the lowest-order perturbation theory. As a result, the conclusion has been made that the $F W$ representation describes some quasi-classic states in the quantum field theories. Both particles and antiparticles are available in these states. Particles, as well as antiparticles, interact with each other. However, there is no interaction of real particles with antiparticles - such interaction is possible only in intermediate (virtual) states. The $F W$ representation modification is required to take into a account real particle/antiparticle interactions. In the papers $[1,2]$ such modification has been made using the symmetry identical to the isotropic spin symmetry owing to invariance of final physical results under change of sings in the mass terms of Dirac Hamiltonian $H_{D}$ and Hamiltonian $H_{F W}$. In the modified Foldy-Wouthuysen representation, real fermions and antifermions can be in two states characterized by the values of the third component of the isotropic spin $S_{f}^{3}= \pm \frac{1}{2}$; real fermions and antifermions interacting with each other must have opposite signs of $S_{f}^{3}$. Quantum electrodynamics in the modified $F W$ representation is invariant under $P$-, $C-, T-$ transformations. Violations of the introduced

1 Instituto de Alta Investigación. Universidad de Tarapacá. Antofagasta No 1520. Arica, Chile. E-mail: htorres@uta.cl 
symmetry of the isotropic spin lead to the corresponding violation of $C P$ - invariance. The standard model in the modified $F W$ representation was formulated in the papers $[1,4]$. It has been shown that formulation of the theory in the modified $F W$ representation doesn't require that Higgs bosons should obligatory interact with fermions to preserve the $S U$ (2)-invariance, whereas all the rest theoretical and experimental implications of the Standard model obtained in the Dirac representation are preserved. In such a case, Higgs bosons are responsible only for the gauge invariance of the boson sector of the theory and interact only with gauge bosons $W_{\mu}^{ \pm}, Z_{\mu}$, gluons and photons.

In the papers mentioned above, the energy representation of Dirac matrices derived by Dirac himself is used:

$$
\alpha^{i}=\left(\begin{array}{cc}
0 & \sigma^{i} \\
\sigma^{i} & 0
\end{array}\right), \beta=\gamma^{0}=\left(\begin{array}{cc}
I & 0 \\
0 & -I
\end{array}\right), \gamma_{5}=\left(\begin{array}{cc}
0 & I \\
I & 0
\end{array}\right), \gamma^{i}=\gamma^{0} \alpha^{i}(1)
$$

Here we propose to change the Foldy-Wouthuysen transformation form by using the chiral representation of Dirac matrices.

$$
\alpha_{c}^{i}=\left(\begin{array}{cc}
\sigma^{i} & 0 \\
0 & -\sigma^{i}
\end{array}\right), \beta_{c}=\gamma_{c}^{0}=\left(\begin{array}{cc}
0 & I \\
I & 0
\end{array}\right), \gamma_{5}=\left(\begin{array}{cc}
I & 0 \\
0 & -I
\end{array}\right), \gamma_{c}^{i}=\gamma_{c}^{0} \alpha_{c}^{i}
$$

The chiral representation (2) is commonly used in the modern gauge field theories and in the Standard Model, in particular.

First consider the structure of equations describing the components of the wave functions $\psi_{D}(x)$ for the two representation of Dirac matrices considered in the paper.

In relations (1), (2) and below the system of units with $\hbar=c=1$ is used; $x, p$, are 4-vectors; the inner product is taken as $x y=x^{\mu} y_{\mu}=x^{0} y^{0}-x^{k} y^{k} \mu=0,1,2,3, k=1,2,3 ; p^{\mu}=i \frac{\partial}{\partial x_{\mu}} ; \sigma^{k}$ are Pauli matrices; $\alpha^{\mu}=\left\{\begin{array}{c}1, \mu=0 \\ \alpha^{i}, \mu=k, k=1,2,3\end{array} ; \psi_{D}(x)\right.$ is the four-component wave function, $\varphi(x), \chi(x), \psi_{R}(x), \psi_{L}(x)$ are the two-component wave functions.

The following operator relations are valid for the free Dirac equation with representation (1):

$$
\begin{aligned}
& p_{0} \psi_{D}(x)=(\vec{\alpha} \cdot \vec{p}+\beta m) ; \psi_{D}(x)=\left(\begin{array}{l}
\varphi(x) \\
\chi(x)
\end{array}\right) ; \\
& \left\{\begin{array}{l}
p_{0} \varphi(x)=\vec{\sigma} \cdot \vec{p} \chi(x)+m \varphi(x) \\
p_{0} \chi(x)=\vec{\sigma} \cdot \vec{p} \varphi(x)-m \chi(x)
\end{array}\right\} ; \\
& \chi=\left(p_{0}+m\right)^{-1} \vec{\sigma} \cdot \vec{p} \varphi ; \\
& \varphi=\left(p_{0}-m\right)^{-1} \vec{\sigma} \cdot \vec{p} \chi ;
\end{aligned}
$$

With representation (2), relation (3) looks like

$$
\begin{aligned}
& p_{0} \psi_{D}(x)=(\vec{\alpha} \cdot \vec{p}+\beta m) ; \psi_{D}(x)=\left(\begin{array}{l}
\psi_{R}(x) \\
\psi_{L}(x)
\end{array}\right) ; \\
& \left\{\begin{array}{c}
p_{0} \psi_{R}(x)=\vec{\sigma} \cdot \vec{p} \psi_{R}(x)+m \psi_{L}(x) \\
p_{0} \psi_{L}(x)=-\vec{\sigma} \cdot \vec{p} \psi_{L}(x)+m \psi_{R}(x)
\end{array}\right\} ; \\
& \psi_{L}(x)=\frac{p_{0}-\vec{\sigma} \cdot \vec{p}}{m} \psi_{R}(x)=\left(p_{0}+\vec{\sigma} \cdot \vec{p}\right)^{-1} m \psi_{R}(x) ; \\
& \psi_{R}(x)=\frac{p_{0}+\vec{\sigma} \cdot \vec{p}}{m} \psi_{L}(x)=\left(p_{0}-\vec{\sigma} \cdot \vec{p}\right)^{-1} m \psi_{L}(x) ;
\end{aligned}
$$

Relations (4) use the operador equality:

$$
p_{0}^{2}=E^{2}=\vec{p}^{2}+m^{2}
$$

Comparison between relations (3) and (4) shows that with the substitution below,

$$
m \leftrightarrow \vec{\sigma} \cdot \vec{p}, \beta \leftrightarrow \gamma_{5}
$$

These relations transform into each other.

The Foldy-Wouthuysen transformations for the energy and chiral representations of Dirac matrices also transform into each other if the substitution (5) is made.

Thus, the general Foldy-Wouthuysen transformation with Dirac matrices in the chiral representation $U_{F W}^{\text {chir }}=\left(U_{F W}^{0}\right)^{\text {chir }}\left(1+\delta_{1}^{\text {chir }}+\delta_{2}^{\text {chir }}++\delta_{3}^{\text {chir }}+\ldots\right)$, as well as the fermion Hamiltonian in the Foldy-Wouthuysen representation

$$
H_{F W}^{c h i r}=\gamma_{5} E+q K_{1}^{c h i r}+q^{2} K_{2}^{c h i r}++q^{3} K_{3}^{c h i r}+\ldots
$$

can be obtained. From the corresponding expressions for $U_{F W}^{e n}, H_{F W}^{e n}$ with Dirac matrices in the energy representation (see $[1,2]$ ) with substitution $m \leftrightarrow \vec{\sigma} \cdot \vec{p}, \beta \leftrightarrow \gamma_{5}$, we have 
the relations

$$
\left\{\begin{array}{c}
p_{0} \psi_{R}(x)=\vec{\sigma} \cdot \vec{p} \psi_{R}(x)+m \psi_{L}(x) \\
p_{0} \psi_{L}(x)=-\vec{\sigma} \cdot \vec{p} \psi_{L}(x)+m \psi_{R}(x)
\end{array}\right\}
$$

\section{CHIRAL APPROACH OF MAXWELL'S EQUATIONS}

Also the relations (6) can be obtained under the chiral approach of Maxwell's Equations where the electric field $\vec{E}$ is parallel to the magnetic field $\vec{H}$ [5], that is $\vec{E}=i \eta \vec{H}$, where $\eta=\sqrt{\mu_{0} / \varepsilon_{0}}$.

If one wanted to describe the hydrogen gas by means of electrodynamics one should start from the firmly established experience that the hydrogen gas may absorb and reemit electromagnetic energy, and that without external intervention there is no indication that the gas to contain electric charges [6-11]. Thus we consider the hypothesis witch visualizes the gas as charge free electromagnetic field as the starting point with the lest number assumptions; and so we try characterize the field by the covariant chiral Maxwell system [5]

$$
\begin{aligned}
& \operatorname{rot} \vec{E}+\mu\left(1+T_{m} \nabla \times\right) \frac{\partial}{c \partial t} \vec{H}=0, \operatorname{div} \varepsilon\left(1+T_{e} \nabla \times\right) \vec{E}=0 \\
& \operatorname{rot} \vec{H}-\varepsilon\left(1+T_{e} \nabla \times\right) \frac{\partial}{c \partial t} \vec{E}=0, \operatorname{div} \mu\left(1+T_{m} \nabla \times\right) \vec{H}=0
\end{aligned}
$$

Here, T is the chiral scalar factor with $\operatorname{div} T_{e, m} \nabla \times \vec{E}(\vec{H})=$ $T_{e, m} \operatorname{div} \nabla \times \vec{E}(\vec{H})=0$, and the condition of charge-free by means of

$$
\operatorname{div} \vec{E}=0 \text { besides } \operatorname{div} \vec{H}=0
$$

Solving the wave equation for $\vec{E}(\vec{H})$ with $T_{e}=T_{m}=T=\hbar / 2 m c$, and by considering we have

$$
\operatorname{rot} \vec{E}(\vec{H})=\mp \frac{1}{T} \vec{E}(\vec{H}) \pm \frac{m c}{\hbar} \vec{E}(\vec{H})
$$

Our hypothesis is that through the multiplication of the matrix Pauli for chiral Maxwell's equations with $\vec{E}=i \eta \vec{H}$, one obtains the chiral Dirac equation (6). Using the algebraic relation [12]

$$
(\hat{\sigma} \cdot \nabla)(\hat{\sigma} \cdot \vec{A})=\operatorname{div} \vec{A}+i \hat{\sigma} \cdot \operatorname{rot} \vec{A}
$$

in equation (10) together with the two div equations (9), transform that system (10) in to

$$
\left(\hat{\sigma} \cdot \nabla \pm i \frac{\omega}{c}\right) \psi_{E}\left(\psi_{H}\right)=-\frac{m c}{\hbar} \psi_{H}\left(\psi_{E}\right)
$$

where $\psi_{E}\left(\psi_{H}\right)=\hat{\sigma} \cdot \vec{E}(\hat{\sigma} \cdot \eta \vec{H})$.

Below the system of units with $\hbar=c=1$ equation (12) is exactly equal to the chiral Dirac equation (6), if $\psi_{E}\left(\psi_{H}\right)=\psi_{R}\left(\psi_{L}\right)$.

To probe this close connection we can obtain the well known normal Dirac equation, we get for $(7,8)$ the equations

$$
\begin{aligned}
& \operatorname{rot} \vec{E}+\mu_{0}\left(1+T_{m} \nabla \times\right) \frac{\partial}{c \partial t} \vec{H}=0, \operatorname{div} \vec{E}=0 \\
& \operatorname{rot} \vec{H}-\varepsilon_{0}\left(1+T_{e} \nabla \times\right) \frac{\partial}{c \partial t} \vec{E}=0, \operatorname{div} \vec{H}=0
\end{aligned}
$$

with $\vec{E} \perp \operatorname{grad} \varepsilon, \vec{H} \perp \operatorname{grad} \mu$. Equations (13) and (14) can be transformed as:

$$
\begin{aligned}
& \mu_{0}\left(1+T_{m} \nabla \times\right) \rightarrow \mu\left(\hbar \omega, m c^{2}\right), \\
& \varepsilon_{0}\left(1+T_{m} \nabla \times\right) \rightarrow \varepsilon\left(\hbar \omega, m c^{2}\right) .
\end{aligned}
$$

So, scalar multiplication of the rot equations in $(10,11)$ by the Pauli-vector, and using the algebraic relation [12] $(\hat{\sigma} \cdot \nabla)(\hat{\sigma} \cdot \vec{A})=\operatorname{div} \vec{A}+i \hat{\sigma} \cdot \operatorname{rot} \vec{A}$ we have

$$
\left\{\begin{array}{l}
(\sigma \cdot \nabla)(\sigma \cdot \vec{H})-\frac{\varepsilon}{c} \frac{\partial}{\partial t}(i \sigma \cdot \vec{E})=0 \\
(\sigma \cdot \nabla)(\sigma \cdot \vec{E})+\frac{\mu}{c} \frac{\partial}{\partial t}(\sigma \cdot \vec{H})=0 \\
\vec{E} \perp \operatorname{grad} \varepsilon, \vec{H} \perp \operatorname{grad} \mu
\end{array}\right\}
$$

Equation (15) can be expressed in terms of in matriz notation this reads

$$
\left.\begin{array}{l}
{\left[\left(\begin{array}{cc}
0 & \sigma \\
\sigma & 0
\end{array}\right) \cdot \nabla-\left(\begin{array}{cc}
\varepsilon 1 & 0 \\
0 & \mu 1
\end{array}\right) \frac{1}{c} \frac{\partial}{\partial t}\right]\left[\begin{array}{c}
i(\sigma \cdot \vec{E}) \\
(\sigma \cdot \vec{H})
\end{array}\right]=0} \\
\vec{E} \perp \operatorname{grad} \varepsilon, \vec{H} \perp \operatorname{grad} \mu
\end{array}\right\}
$$


Denoting the quantity on witch the differential operators act by $\psi_{D}$, that is

$$
\left[\begin{array}{c}
i(\sigma \cdot \vec{E}) \\
(\sigma \cdot \vec{H})
\end{array}\right]=\left(\begin{array}{cc}
i E_{3} & i H_{-} \\
i E_{+} & -i E_{3} \\
H_{3} & H_{-} \\
H_{+} & -H_{3}
\end{array}\right)=\left(\begin{array}{cc}
\Psi_{1}^{\prime} & \Psi_{1}^{\prime \prime} \\
\Psi_{2}^{\prime} & \Psi_{2}^{\prime \prime} \\
\Psi_{3}^{\prime} & \Psi_{3}^{\prime \prime} \\
\Psi_{4}^{\prime} & \Psi_{4}^{\prime \prime}
\end{array}\right)
$$

with $X_{ \pm}=X_{1} \pm i X_{2}$ and considering the well-known connection

$$
\left(\begin{array}{ll}
0 & \sigma \\
\sigma & 0
\end{array}\right)=\gamma \equiv \alpha
$$

between the Pauli and Dirac matrices, we get for (13) system

$$
\left\{\begin{array}{l}
{\left[\gamma \cdot \nabla-\left(\begin{array}{cc}
\varepsilon 1 & 0 \\
0 & \mu 1
\end{array}\right) \frac{1}{c} \frac{\partial}{\partial t}\right] \Psi_{D}=0} \\
\vec{E} \perp \operatorname{grad} \varepsilon, \vec{H} \perp \operatorname{grad} \mu
\end{array}\right\}
$$

Here one has to bear in mind that each of both columns matrix (14) that is

$$
\Psi_{D}=\left(\begin{array}{c}
i E_{3} \\
i E_{1}-E_{2} \\
H_{3} \\
H_{1}+i H_{2}
\end{array}\right) \text { and } \Psi_{D}=\left(\begin{array}{c}
i E_{1}+E_{2} \\
-i E_{3} \\
H_{1}-i H_{2} \\
-H_{3}
\end{array}\right)
$$

Independently represent a system of functions solving (16). From this, a separation of the time dependence according to

$$
\Psi_{D}=\psi_{D} e^{-i \omega t}
$$

finally yields the amplitude equation

$$
\left\{\gamma \cdot \nabla-i \frac{\omega}{c}\left(\begin{array}{cc}
\varepsilon 1 & 0 \\
0 & \mu 1
\end{array}\right)\right\} \Psi_{D}=0
$$

If we use equation (13) in (22), it's agreement with the Dirac amplitude equation

$$
\gamma \cdot \nabla \Psi+\frac{i}{c \hbar}\left(\begin{array}{cc}
\hbar \omega+m c^{2} & 0 \\
0 & \hbar \omega-m c^{2}
\end{array}\right) \Psi_{D}=0
$$

is complete. Now normalizing eq. (23) with $\hbar=1, c=1, \gamma \cdot \nabla=\alpha \cdot p$, we can write as

$$
p_{0} \psi_{D}(x)=(\vec{\alpha} \cdot \vec{p}+\beta m) ; \psi_{D}(x)
$$

And the transformation to a chiral Dirac equation is trivial by using relation (5).

The equations (20) as well as (21) or (22), show in addition that the electrodinamical and the wave mechanical field component are connected by simple linear relation, the same holding true for the refraction $(\varepsilon, \mu)$ in relation to the scalar $\mathrm{T}$.

This isomorphism can be checked easily and directly because the eight Eq. $(10,11)$ may be combined into two systems of four equations each, in the following way:

$$
\left\{\begin{array}{l} 
\pm i(\operatorname{rot} \vec{H})_{3} \mp i c^{-1} \varepsilon \dot{E}_{3}+\operatorname{div} \vec{H}=0 \\
\pm i(\operatorname{rot} \vec{H})_{1} \mp i c^{-1} \varepsilon \dot{E}_{1}-(\operatorname{rot} \vec{H})_{2}+c^{-1} \varepsilon \dot{E}_{2}=0 \\
\pm i d i v \vec{E}-(\operatorname{rot} \vec{E})_{3}-c^{-1} \mu \dot{H}_{3}=0 \\
\pm i(\operatorname{rot} \vec{E})_{2} \mp i c^{-1} \mu \dot{H}_{2}-(\operatorname{rot} \vec{E})_{1}+c^{-1} \mu \dot{H}_{1}=0
\end{array}\right\}
$$

Inserting here the first or second wave function of (21) into the first system (upper signs) or the second one (lower signs), respectively, the wave functions of (20) ends up immediately, in both cases and we are back to Dirac again

\section{CONCLUSION}

Using a chiral representation of the Foldy-Wouthuysen transformation for the Dirac equation we show that the same result can be obtained with a chiral electrodynamics using the matrix Pauli.

With this we proof the theorem that waves mechanic of quantum particle represents a specialized electrodynamics. The result seems unambiguous and incompatible with the current doctrine which rest on a particle interpretation.

\section{REFERENCES}

[1] V.P. Neznamov. Physics of Elementary Particles and Atomic Nuclei (EPAN). Vol. $37 \mathrm{~N}^{\circ}$ 1. 2006.

[2] V.P. Neznamov. Voprosy Atomnoi Nauki I Tekhniki. Ser: Teoreticheskaya I Prikladnaya Fizika. Issues 1-2, p. 41, hep-th/0411050. 2004. 
[3] L.L. Foldy and S. A. Wouthuysen, Phys. Tev 78, 29. 1950.

[4] V.P. Neznamov. Hep-th/0412047. 2005.

[5] H. Torres-Silva and M. Zamorano Lucero. Chiral Electrodynamic. URLs: http://www.chiral.cl

[6] J.R. Oppenheimer. Phys. Rev. Vol. 38, p. 725. 1931.

[7] H.E. Moses. Sup. Nuovo Cimento. Serie X. Vol. 7. $\mathrm{N}^{\circ} 1.1958$.

[8] T. Ohmura Prog. Theor. Phys. Vol. 16, p. 684. 1956.
[9] S.N. Gupta. Theory of longitudinal photons in quantum electrodynamics. Proc. Phys. Soc. Vol. 63, pp. 681-691. 1950.

[10] F. Reines and W. H. Sobel, Test of the Pauli Exclusion Principle for Atomic Electrons, Phys. Rev. Lett. Vol. 32, pp. 954. 1974.

[11] W. Heitler Quantum Theory of Radiation, $2^{\text {nd }}$ Ed., Oxford University Press, Oxford, p. 1. 1944.

[12] H. Sallhofer. "Maxwell Dirac isomorphism". Z. Naturforsch. Vol. 41 a, p 1067. 1986. 